\title{
Transplantation in highly HLA-sensitized patients: challenges and solutions
}

\author{
This article was published in the following Dove Press journal: \\ Transplant Research and Risk Management \\ 26 September 2014 \\ Number of times this article has been viewed
}

\author{
Irene K Kim \\ Ashley Vo \\ Stanley C Jordan \\ Transplant Immunotherapy Program, \\ Comprehensive Transplant Center, \\ Cedars-Sinai Medical Center, \\ Los Angeles, CA, USA
}

Correspondence: Irene K Kim Comprehensive Transplant Center, Cedars-Sinai Medical Center, 8635 W 3rd St Suite 590 W, Los Angeles, CA 90048 , USA

$\mathrm{Tel}+\mid 310423264$ |

Email irene.kim@cshs.org

\begin{abstract}
Despite better understanding of the impact of development of the human leukocyte antigen (HLA) antibody and numerous advancements in immunosuppressive therapy, the ability to successfully transplant highly sensitized patients remains a significant challenge. As the percentage of the waiting list becomes increasingly populated with highly sensitized patients, there is a growing demand for effective strategies to manage these patients. Over the past 20 years, desensitization therapies have been modified and developed, and are mainly utilized at transplant centers that have developed expertise. In addition, recognition that the highly sensitized patient population is disadvantaged on the transplant waiting list has led to recent changes in national kidney allocation policy. Furthermore, creative strategies, such as enrollment of sensitized patients into paired kidney exchange programs, have been developed to find compatible matches for these patients. The goal of this article is to address some of the specific challenges related to transplanting the highly sensitized patient at a high-volume transplant center with experience in desensitization and to review established and emerging solutions to help this patient population.
\end{abstract}

Keywords: human leukocyte antigen, antibodies, desensitization, high-dose intravenous immunoglobulin, rituximab

\section{Introduction}

Renal transplantation remains the treatment of choice for patients suffering from end-stage renal disease, showing clear survival benefits and reduced morbidity. ${ }^{1,2}$ Unfortunately, the demand far exceeds the supply of available organs for transplant, rendering many patients without this life-saving treatment. The number of patients on the deceased donor kidney waiting list has now exceeded 100,847 as of June 12, 2014, while the number of kidney transplants performed in the USA per year has remained fairly constant at about 15,000 transplants per year. ${ }^{3}$

Among the most vulnerable populations awaiting a kidney transplant are patients on the waiting list who are highly human leukocyte antigen (HLA)-sensitized. Patients become highly sensitized after exposure to HLA through blood transfusions, pregnancy, or prior transplantation. It is estimated that nearly $30 \%$ of patients on the deceased donor waiting list are considered sensitized, defined as a panel-reactive antibody (PRA) of $30 \%-80 \%{ }^{3}$ An additional cohort of patients are considered broadly sensitized with a PRA $>80 \%$. HLA-sensitized patients have emerged as one of the greatest challenges in transplant surgery, affecting not only patients on the kidney waiting list, but also other solid organ transplant recipients, including heart, lung, and bowel transplant recipients. 
There are two major challenges associated with transplanting HLA-sensitized patients. Firstly, the presence of HLA antibodies often poses a roadblock to transplantation. Patients with high anti-HLA donor-specific alloantibodies have a relative contraindication to transplantation, especially in the presence of a positive complement-dependent cytotoxicity crossmatch, which leads to hyperacute rejection and allograft loss. ${ }^{4,5}$ Second, the presence and development of alloantibody can lead to antibody-mediated rejection and transplant graft loss once a patient has been transplanted. It has long been established that T-cell mediators are important in allograft rejection and it is now established that B-cells and alloantibodies also play a critical role in allograft injury., ${ }^{4,5}$ Donor-specific antibodies (DSA) are now implicated in a complex array of antibody-mediated rejection (ABMR), including a particularly difficult form of chronic rejection termed transplant glomerulonephropathy. ${ }^{6,7}$ This article aims to discuss well established and emerging solutions in combating the presence of alloantibody and overall approach to the highly sensitized patient at a high-volume kidney transplant center.

\section{Solutions}

The most significant advance in successfully treating highly sensitized patients has been the development of desensitization therapy. These emerging protocols have had success in enabling transplantation in HLA-sensitized patients and also in treating antibody-mediated rejection. In addition, additional drug targets against antibody-mediated rejection are also being developed, showing preliminary success in treating this population. Lastly, policy changes in organ allocation and other creative solutions have been developed in order to successfully transplant these patients.

\section{Desensitization}

Due to the demand for therapy directed against humoral immunity and the growing number of HLA-sensitized patients awaiting transplantation, desensitization protocols began to emerge in the late 1990s. The goals of desensitization therapy are clear, ie, to clear or decrease the presence of circulating alloantibody and to prevent the onset of antibodymediated rejection. Plasma exchange (PLEX) and drugs that modify B-cells and antibodies have been developed and used for desensitization therapy. PLEX is a minimally invasive procedure that can remove immunoglobulin from a patient's sera and has been shown to lower HLA-specific antibody levels in many clinical settings. ${ }^{8,9}$

Intravenous immunoglobulin (IVIG) is a complex preparation derived from pooled human plasma and has been used in the treatment of primary immunodeficiency disorders for many years. The mechanism of action for this drug is not completely understood, but its effects appear to be broadacting. It has long been viewed as having a neutralizing effect on circulating autoantibodies and alloantibodies; however, IVIG also has the ability to interact with multiple cell types involved in immune activation, both innate and adaptive. ${ }^{10,11}$ It also appears to play a role in inhibiting complement activation, which is a recognized factor in ABMR. ${ }^{12}$

Currently, there are two well established desensitization protocols that have gained wide acceptance. The first uses low-dose IVIG with plasma exchange (PLEX) and was first demonstrated by the transplant group at Johns Hopkins. This protocol has been used successfully in ABO-incompatible and positive crossmatch (+CMX) kidney transplantation. ${ }^{13,14}$ The second regimen, which was developed by our group at Cedars-Sinai Medical Center, uses high-dose IVIG in the form of $2 \mathrm{~g} / \mathrm{kg}$ in multiple doses. The latter protocol has been used to successfully desensitize both living donor +CMX recipients and HLA-sensitized candidates on the deceased donor waiting list. ${ }^{15-17}$

Rituximab is a monoclonal antibody against CD20, a membrane-bound protein found on B-cells and is known to be efficacious in the treatment of B-cell malignancies and certain autoimmune disorders. CD20 is highly expressed on B-cells but expression is lost with transition to plasma cells. B-cell depletion using rituximab is often used in conjunction with both of these therapies and has been coined as providing a "medical splenectomy". We will further elaborate on the basic principles of these protocols with their respective advantages and disadvantages.

\section{IVIG/plasma exchange}

The IVIG/PLEX regimen uses a lower dose of IVIG $(100 \mathrm{mg} / \mathrm{kg})$ and is known to be effective as rescue therapy in acute humoral rejection and also as a desensitization strategy for transplantation in $+\mathrm{CMX}$ recipients. However, this therapy is limited to live donor kidney transplantation due to the rebound phenomenon of DSA within days of discontinuing therapy. Therefore, when timing of transplantation is unknown, such as in patients on the deceased donor waiting list, IVIG/PLEX is not a feasible option.

In the first series, published by Montgomery et al, ${ }^{13}$ IVIG/ PLEX was shown to be successful in the pre-emptive removal of DSA in seven sensitized patients prior to living donor kidney transplantation. Four +CMX patients (three with flow $+\mathrm{CMX}$, one with cytotoxic +CMX) underwent successful pre-emptive desensitization. Patients received tacrolimus 
and mycophenolate mofetil on the first day of PLEX, which consisted of replacement of 1-1.5 plasma volumes using either 5\% albumin or fresh frozen plasma. Immediately following PLEX, patients received $100 \mathrm{mg} / \mathrm{kg}$ standard IVIG or Cytogam ${ }^{\circledR}$. At the time of transplant, patients received induction therapy with anti-interleukin (IL)-2 receptor antibody and steroids. An additional three patients received this regimen for rescue therapy after established acute humor rejection following living donor kidney transplantation. The overall mean creatinine was $1.4 \pm 08 \mathrm{mg} / \mathrm{dL}$ with a mean follow-up of $58 \pm 40$ weeks.

\section{High-dose IVIG}

The rationale for high-dose IVIG stems from the observation that higher doses (1-2 g/kg per dose) were needed to achieve immunomodulatory effects compared with patients receiving IVIG in immunodeficiency. To our knowledge, our group was the first to report the findings of a randomized, multicenter, placebo-controlled trial of desensitization therapy versus dialysis alone for patients awaiting kidney transplantation (NIH-IGO2 study, 1997-2002). Between 1997 and 2000, 101 adult patients with end-stage renal disease who were highly sensitized received either high-dose IVIG $(2 \mathrm{~g} / \mathrm{kg})$ or placebo. Patients receiving high-dose IVIG had a statistically significant reduction in anti-HLA antibody levels ( $P=0.004$, IVIG versus placebo). Additionally, improved rates of transplantation were observed in the treatment group, where 16 IVIG patients $(35 \%)$ versus eight placebo $(17 \%)$ patients were transplanted $(P=0.031)$. Compared with patients who did not receive desensitization therapy, patients who underwent IVIG desensitization had a mean wait time of 4.8 years versus 10.3 years in the placebo group $(P=0.02)$ at a mean follow-up of 3 years. Lastly, there was improved allograft survival at 3 years despite more episodes of acute rejection observed in the IVIG group. ${ }^{16}$

The approach developed at our center, based on the above trial, utilizes four doses at monthly intervals of highdose IVIG (2 g/kg, maximum dose $140 \mathrm{~g})$ until a negative or acceptable crossmatch is obtained $(<225$ mean channel shifts by flow cytometry crossmatch). We have implemented this protocol for patients who are highly sensitized and have been on the United Network for Organ Sharing (UNOS) waiting list for $>5-7$ years, those who have a PRA $>30 \%$, and those who receive frequent allograft offers but continually have a $+\mathrm{CMX}$.

The one-year, 3-year, and 5-year outcomes for patients undergoing desensitization with high-dose IVIG was found to be comparable, with reported UNOS graft outcomes for patients with PRAs of $0 \%-9 \%$ and $10 \%-79 \%$ versus the poorer outcomes for patients with PRAs of $>80 \%$. Graft failure after desensitization was mainly attributed to acute rejection (defined as rejection within the first year), whereas late failures were due to noncompliance or death with a functioning graft. ${ }^{17}$ Recent reports have suggested that plasmapheresis-based protocols are somewhat more effective than high-dose IVIG protocols at reducing DSA. ${ }^{18}$ In practice, this creates a dilemma as PLEX-based protocols are difficult to implement for patients awaiting transplantation on the deceased donor waiting list.

\section{IVIG \pm rituximab}

The utility of rituximab alone as a desensitizing agent has been questioned after the observation that it seemed to have relative inefficacy at decreasing DSA in highly sensitized patients. ${ }^{19,20}$ For patients who were non-responders to highdose IVIG or who developed high-titer anti-HLA antibodies, our group at Cedars-Sinai Medical Center sought to investigate the effect of rituximab with an established desensitization protocol. Furthermore, this protocol used only two doses of IVIG with rituximab and was found to require less time, reducing the time of desensitization from 16 weeks to 4-5 weeks of therapy. Two weekly doses of rituximab were sandwiched in between high-dose IVIG administration. This study demonstrated an increased ability to transplant highly sensitized patients $(80 \%)$ with excellent patient and allograft survival and also proved to be less costly. ${ }^{21}$

The efficacy of desensitization with IVIG + rituximab was further analyzed at our center. Between July 2006 and December 2011, 207 broadly sensitized patients (PRA >80\%) received desensitization with IVIG + rituximab. Of the 207 patients, 146 (71\%) patients were transplanted, and at 48 months post-transplant, patient and graft survival by Kaplan-Meier were $95 \%$ and $87.5 \%$, respectively. Compared with matched HLA-sensitized patients with PRA $>80 \%$ on the UNOS wait list, the mean rate of transplantation was significantly higher for patients undergoing desensitization $(67 \%$ versus $10 \%$ ) over a 3 -year period. Linear regression analysis showed a $5.5 \%$ increase in deceased donor transplant rates per year compared with a $0.4 \%$ increase in HLA-sensitized patients remaining on the UNOS wait list. ${ }^{22}$

\section{Challenges with desensitization Need for HLA support}

The implementation of a desensitization program requires a collaborative team approach with expertise in HLA antigen laboratory support and should not be undertaken without 
this expertise. Determination of acceptable crossmatches is crucial to identifying patients eligible for transplantation after desensitization therapy. Therefore, the goal of desensitization therapy is not necessarily to reach a negative crossmatch, but rather reduce DSA to an acceptable level for transplantation and to accurately characterize the specificity and strength of HLA antibodies. ${ }^{23,24}$ Approximately $63 \%$ of patients were transplanted at our center, with a positive flow crossmatch with the high-dose IVIG and rituximab regimen. The determination of acceptable DSA levels allowing successful deceased and living donor transplantation after desensitization was studied by our center. Our experience showed that patients with DSA more than $10^{5}$ standard fluorescence intensity/5,000 mean fluorescent intensity and T-cell flow crossmatches more than 225 mean channel shifts were more susceptible to antibody-mediated rejection and required more intensive antibody monitoring post transplantation. ${ }^{25,26}$ The practice at our center is to input unacceptable or avoid antigens with standard fluorescence intensity $>200,000$ (mean fluorescent intensity $>10,000$ ) or any antigen with $\mathrm{C} 1 \mathrm{q}$ positivity, an assay that detects HLA antibodies that can fix complement and identify DSA that are clinically harmful. ${ }^{26}$

\section{Cost of IVIG therapy in transplant patients}

Concerns about the economics and justification of the cost for established desensitization protocols have been raised. IVIG is an expensive preparation and may not be easily approved by insurers and hospitals. For example, the four-dose course of the high-dose IVIG protocol for a $70 \mathrm{~kg}$ patient is estimated to be nearly $\$ 28,090$ per patient. To justify the cost of this important therapy, one must compare the cost-benefit analysis of desensitization therapy leading to transplantation with the cost of patients remaining on hemodialysis. According to US Renal Data System 2008 data and assuming inflation costs of $3.43 \%$ per year, the estimated cost of maintaining a patient on hemodialysis was $\$ 84,639$ per year and for peritoneal dialysis, the cost accrued was \$53,327 per year in 2006 . The cost of transplantation was estimated to be $\$ 92,799$ per patient and for annual maintenance immunosuppression was $\$ 17,091$ per year. ${ }^{27,28}$ Therefore, over a 5-year period, a patient successfully transplanted, even when factoring in the cost of desensitization, costs roughly $\$ 155,071$, compared with $\$ 423,195$ to maintain a patient on 5 years of hemodialysis, ie, $\mathrm{a}>\$ 250,000$ difference. ${ }^{22}$

It should also be noted that in examining the cost efficacy of desensitization, there were also "non-responders" to desensitization therapy who remained on dialysis. In the series published by Vo et al, 61/207 (29\%) patients who were desensitized with IVIG + rituximab remained on dialysis. Additionally, approximately eleven patients (7.5\%) experienced graft loss after desensitization therapy followed by transplantation. However, in patients who had no graft loss, the cost of desensitization, transplantation, and immunotherapy was estimated to be $\$ 18,911$ per patient per year compared with $\$ 84,639$ per patient per year for patients who received desensitization and were also not transplanted. Given that nearly $70 \%$ of patients receiving therapy in this series were transplanted, there seems to be an overall cost benefit, even factoring in patients who were non-responders to therapy. ${ }^{22}$

Analysis of the IG02 study ${ }^{16}$ estimated that the calculated cost savings with IVIG were approximately $\$ 300,000$ per patient transplanted versus those remaining on hemodialysis for 5 years. This estimate was further supported by the US Renal Data System, analysis of which demonstrated the cost savings to Medicare for highly sensitized patients successfully transplanted compared with patients remaining on dialysis. ${ }^{22,29,30}$ A meta-analysis by the Canadian Blood Services also concluded that sensitized patients receiving IVIG had better survival and decreased mortality than dialysis patients. ${ }^{28}$

\section{Desensitization and risk of infection and malignancy}

Another concern with desensitization is the administration of more immunosuppression, which may lead to higher rates of infection and malignancy, especially with rituximab administration. At some centers, infection was the leading cause of death in patients receiving desensitization therapy, and problems with BK nephropathy have also been observed. ${ }^{31,32}$ We retrospectively reviewed our experience with IVIG + rituximab desensitization therapy in both highly sensitized patients and patients receiving therapy for $\mathrm{ABO}$-incompatible transplants. The risk of infection with desensitization was compared with patients who did not receive desensitization, where all patients received antiviral and antimicrobial prophylaxis at the time of transplantation. There were no differences in rates of bacterial ( $34.7 \%$ versus $39.1 \%$ ), viral (21.8\% versus $25.1 \%$ ), fungal (5.9\% versus $5.2 \%$ ), or serious infections (22.9\% versus $25.5 \%$ ) in both the desensitization and non-desensitization groups. This analysis concluded that rituximab did not increase the risk of infection. ${ }^{32}$

Polyomavirus BK viremia (BKV) was found to be higher in patients receiving IVIG plus rituximab compared with non-sensitized transplant recipients (18 versus eleven 
patients, $P=0.09$ ), although not statistically significant in our initial analysis. Barbosa et al conducted a more comprehensive analysis of $\mathrm{BKV}$ in patients receiving desensitization with IVIG and rituximab $(n=187)$ and compared outcomes with non-desensitized patients $(n=248)$ at our center. BKV was observed in $20 \%$ of the desensitized patients and $10 \%$ of the non-desensitized group $(P<0.001)$ by 2 years post transplant; however, there was no significant difference in $\mathrm{BKV}$-associated nephropathy or graft loss in the two groups. ${ }^{33}$

The risk of malignancy, particularly skin cancers and lymphoproliferative disorders, with long-term immunosuppression is a known adverse event in transplantation. The risk of cancer in patients who also receive desensitization is not known; however, the incidence does not appear to be any greater than in transplant patients not receiving desensitization therapy. ${ }^{32,34}$

\section{Surgical solutions: splenectomy as rescue therapy}

Splenectomy has been historically used as pre-emptive treatment for patients undergoing ABO-incompatible transplants or patients with a high HLA antibody load. Although no longer widely used in desensitization, its role in rescue therapy may be helpful. Approximately $30 \%$ of $+\mathrm{CMX}$ patients have a higher rate of ABMR, which is usually mild and responsive to further IVIG/PLEX treatment. Patients with severe ABMR occurring after the first week post transplant have been successfully rescued by splenectomy in conjunction with further medical therapy at our center. Several other centers have also reported salvage of allografts and immediate return of renal function following splenectomy for severe ABMR. ${ }^{35,36}$ This surgical intervention should be considered in difficult cases of severe ABMR.

A medical alternative to splenectomy for salvage therapy has utilized eculizumab, which is a humanized monoclonal antibody against $\mathrm{C} 5$, the terminal portion of the complement cascade. Recently, our center has attempted a new strategy using eculizumab for salvage therapy in severe ABMR that manifested with thrombotic microangiopathy after transplantation (unpublished data). We were able to salvage $2 / 2$ grafts with thrombotic microangiopathy using PLEX + eculizumab.

\section{Surgical considerations surrounding desensitization}

Given that many HLA-sensitized patients have received prior renal allografts, our center has had a somewhat unique surgical experience as a high-volume desensitization center.
Patients who have received two previous allografts are evaluated by computed tomography angiogram to determine vascular patency and the surgical approach. Transplant nephrectomy of a previous allograft is rarely indicated and performed. Placement of a new allograft can be either retroperitoneal or intra-abdominal according to the surgeon's discretion. In addition, because HLA-sensitized patients often have long wait times, sometimes greater than 10 years on dialysis, thrombotic complications of dialysis access can jeopardize surgical eligibility for transplantation.

Patients often receive PLEX as part of desensitization and undergo plasmapheresis via tunneled hemodialysis access catheters. We have experienced some clotting issues with these temporary catheter placements, which has identified some patients with possible hypercoagulable states prior to transplantation. Our approach for any patient with clotting access as part of desensitization therapy has been to obtain a hypercoagulable work-up and in some cases, has led to heparinization of the patient during transplantation.

\section{Changes in organ allocation policy}

Despite prioritization points on the deceased organ transplant list, fewer than $15 \%$ of highly sensitized patients are transplanted per year. The UNOS implements the nation's transplant organization known as the Organ Procurement and Transplantation Network, and strives to continually optimize allocation policy to maximize organ utilization and patient access to transplantation. On June 25, 2013, the UNOS/Organ Procurement and Transplantation Network approved significant revisions to the kidney allocation policy, including policies benefitting patients waiting on the transplant list who are "biologically disadvantaged". The revised policy, which is projected to take effect December 2014, will enhance access to transplantation for patients with less common blood types and patients who are highly sensitized. The current kidney allocation policy assigns a set amount of points for prioritization on the waiting list based on waiting time, sensitization status, antigen mismatch, and pediatric patients. Currently, there is a maximum of four points given to patients identified with immunologic sensitivity, measured by the calculated panel reactive antibody (CPRA) score. The policy change seeks to replace the current scoring system with a sliding scale of points dependent on degree of sensitization as reported by CPRA. For example, patients with a CPRA of 98 will receive approximately 24 additional points, whereas patients with 100\% CPRA will be given 202 points, effectively giving highly sensitized patients national priority. ${ }^{37,38}$ 
These policy changes may provide rare opportunities for highly sensitized patients to receive an acceptable deceased donor match while on the waiting list. As these changes have yet to be implemented, it is unclear what the effect will be on successfully transplanting highly sensitized patients. Preliminary mathematical modeling has shown a projected improvement in post-transplant survival and access for highly sensitized patients. ${ }^{38}$

\section{Kidney paired donation programs}

Patients fortunate enough to have a potential living donor can be transplanted sooner; however, highly sensitized patients, despite desensitization therapy, are often found to be incompatible with their willing living donors. Kidney paired donation has emerged as a creative option to match more immunologically compatible recipients and donors. There exist several kidney paired donation program options, the largest being the National Kidney Registry database, although single center options also exist, including Johns Hopkins and Methodist San Antonio. One strategy to transplant highly sensitized patients relies on a combination of desensitization and kidney paired donation enrollment, which increases the pool of potentially compatible living donors, thereby increasing the odds that a highly sensitized patient can find a compatible match. ${ }^{39}$

Stanford University recently published their experience with five highly sensitized patients, all with CPRA scores of $100 \%$, who were entered into paired donation programs and received desensitization therapy. Three of these five patients were successfully transplanted via kidney paired donation, while the remaining two patients were ultimately transplanted from the deceased donor waiting list. Patients were desensitized with monthly high-dose IVIG $(2 \mathrm{~g} / \mathrm{kg})$, rituximab $\left(375 \mathrm{mg} / \mathrm{m}^{2}\right)$ after four doses of IVIG, and plasmapheresis followed by bortezomib (Velcade ${ }^{\circledR}$ ), a proteasome inhibitor that induces cellular apoptosis of normal plasma cells, at $1.3 \mathrm{mg} / \mathrm{m}^{2}$ in non-responders to desensitization therapy. An acceptable crossmatch was defined as T-cell and B-cell flow crossmatch with a median flow channel shift of $\leq 200$ after adjusting for the presence of autoantibodies. Two of the three patients transplanted via kidney paired donation suffered borderline acute cellular rejection (C4d negative) and all three patients did not develop DSA up to 22 months post transplant. ${ }^{40}$

At our program, we have also succeeded in transplanting three HLA-sensitized patients using a similar approach of desensitization and enrollment into kidney paired donation. Between 2013 and 2014, two patients with willing living donors and an altruistic donor enabled two paired exchanges, leading to transplantation of three HLA-sensitized patients at our center. The two HLA-sensitized recipients with living donors were found to be persistently incompatible with their potential donors due to DSA refractory to desensitization. One of these chains ended with the transplantation of a HLAsensitized patient on our deceased waiting list, enabling us to successfully transplant three HLA-sensitized patients. Two of the recipients were also ABO-incompatible with their donors, but the exchange donors had more suitable DSA profiles with a higher probability for response to desensitization.

\section{Emerging therapies}

Newer therapies aimed at B-cells and antibodies have emerged from the autoimmunity market. These also hold potential for clinical application in modifying alloantibodies and alloreactive B-cells. Some of these new drug targets are currently being investigated in the transplant population, whereas others have theoretical benefit.

\section{Alemtuzumab}

Alemtuzumab (Campath ${ }^{\circledR}$ ) is a monoclonal antibody that binds to CD52, targeting the destruction of mature lymphocytes, and has been used as standard immunosuppressive induction therapy. It offers a potential additive immunosuppressive effect on B-cells that may prove more effective in HLA-sensitized patients, reducing the rates of ABMR. In a non-randomized study published by our group, alemtuzumab did not result in reduced ABMR rates post transplant compared with antithymocyte globulin induction therapy and daclizumab. There were similar rates of infection observed and overall, seemed to be more cost-effective. ${ }^{41}$

\section{Bortezomib}

Bortezomib (Velcade ${ }^{\circledR}$ ) has also been used as a plasma celldepleting agent and has been used successfully in the treatment of ABMR. Everly et al effectively treated six patients with ABMR and acute cellular rejection with bortezomib, with resolution of rejection and marked reduction in DSA levels. ${ }^{42}$ More data are needed to determine the efficacy of bortezomib as a desensitization agent in a randomized controlled trial, but it may hold promise as a depleting agent in nonmalignant plasma cells.

\section{Anti-IL-6 receptor therapy}

IL-6 is a pleiomorphic cytokine with broad-ranging effects and has been found to play a major role in autoimmune 
disorders. Tocilizumab (Actemra ${ }^{\circledR}$ ) is a monoclonal human antibody directed against the IL-6 receptor, competitively inhibiting IL-6, and has been approved by the US Food and Drug Administration for treatment of autoimmune disorders such as rheumatoid arthritis and Castleman disease. In these inflammatory diseases, administration of tocilizumab has led to significant reductions in serum immunoglobulin $G$ and A levels and a reduction in inflammatory response.

The role of IL-6 and IL-6 receptor blockade in transplantation is largely unknown. We have recently published our experience with anti-IL-6 receptor blockade in an animal transplantation model, demonstrating that IL-6 inhibition leads to a reduction of de novo DSA response after allosensitization. ${ }^{43,44}$ In addition, we are completing an openlabel Phase I/II clinical trial examining the use of tocilizumab in highly sensitized patients who have failed desensitization with high-dose IVIG and rituximab (ClinicalTrials.gov identifier NCT01594424). Our initial results with this trial have been encouraging, but further studies are needed to determine the effects of this new drug.

\section{Other anti-CD20 monoclonal antibodies}

The success of the anti-CD20 monoclonal antibody, rituximab, in treating B-cell malignancies, autoimmune diseases, and patients undergoing transplantation has led to interest in the development of other drug targets against the CD20 receptor. These drugs include ocrelizumab (humanized anti-CD20, Genentech/Roche, South San Francisco, CA, USA), ofatumumab (humanized anti-CD20, Genmab, Copenhagen, Denmark) and veltuzumab (humanized antiCD20, Immunomedics, Morris Plains, NJ, USA). Most of these drugs are currently in Phase II/III clinical trials for treatment of autoimmune diseases and leukemia. There are currently no known investigational trials of these medications in transplantation. ${ }^{45}$

\section{Anti-CD 19 and anti-CD22}

Other B-cell-specific membrane receptors have been targets for drug therapy. CD19 is present on B-cells from the earliest cellular lineage during B-cell development but is lost in maturation to plasma cells. MD1342 is a human monoclonal directed against CD19 (Medarex, Princeton, NJ, USA) and a Phase I/II clinical trial using this drug in patients with rheumatoid arthritis was suspended. Although there is a theoretical use of this drug in antibody-mediated diseases, there has been no investigation of its usage in transplant patients. ${ }^{46} \mathrm{CD} 22$ is a cell surface protein found on mature and some immature B-cells. Epratuzumab (Immunomedics) is a humanized anti-CD22 antibody that depletes about $35 \%$ of the total B-cell population. This drug is currently in Phase III studies of patients with systemic lupus erythematosus and appears to be well tolerated. Again, the effect of this medication in transplant and highly sensitized patients is unknown.

\section{Conclusion}

Protocols using either high-dose IVIG or plasmapheresis with low-dose IVIG are safe and viable options for highly sensitized patients awaiting transplantation. Implementation of these protocols requires expertise in HLA laboratory assessment and pharmacologic experience. Furthermore, PLEX services and rapid analysis of kidney biopsies are necessary for optimizing therapy. All these factors should be considered before embarking on a desensitization program. New allocation policies and kidney paired exchange could further enable successful transplantation in the highly sensitized population. Major surgical concerns for transplanting highly sensitized patients include the overall surgical suitability of patients often on dialysis for more than a decade, surgical access due to previous transplants, and risk for thrombotic complications. Lastly, new and emerging therapies in development could hold further promise in treating these patients.

\section{Disclosure}

The authors report no conflicts of interest in this work.

\section{References}

1. Evans RW, Manninen DL, Garrison LP Jr, et al. The quality of life of patients with end-stage renal disease. $N$ Engl J Med. 1985;312: 553-559.

2. Port FK, Wolfe RA, Mauger EA, Berling DP, Jiang K. Comparison of survival probabilities for dialysis patients vs cadaveric renal transplant recipients. JAMA. 1993;270:1339-1343.

3. The 2012 Annual Data Report [webpage on the Internet]. Minneapolis: US Department of Health \& Human Services, Scientifc Registry of Transplant Recipients. Available from: http://srtr.transplant.hrsa.gov/ annual_reports/2012. Accessed September 4, 2014..

4. Kissmeyer-Nielsen F, Olsen S, Peterson VP, Fjeldborg O. Hyperacute rejection of kidney allografts, associated with pre-existing humoral antibodies against donor cells. Lancet. 1966;2:662-665.

5. Patel R, Terasaki PI. Significance of the positive crossmatch test in kidney transplantation. N Engl J Med. 1969;280:735-739.

6. Platt JL. Antibodies in transplantation. Discov Med. 2010;10: $125-133$.

7. Lee PC, Terasaki PI, Takemoto SK, et al. All chronic rejection failures of kidney transplants were preceded by the development of HLA antibodies. Transplantation. 2002;74:1192-1194.

8. Taube D, Williams D, Cameron J. Renal transplantation after removal and prevention of resynthesis of HLA antibodies. Lancet. 1984;1: 824-828.

9. Hodge EE, Klingman LL, Koo AP, et al. Pretransplant removal of anti-HLA antibodies by plasmapheresis and continued suppression on cyclosporine-based therapy after heart-kidney transplant. Transplant Proc. 1994;26:2750-2751. 
10. Tha-In T, Bayry J, Metselaar HJ, Kaveri SV, Kwekkeboom J. Modulation of the cellular immune system by intravenous immunoglobulin. Trends Immunol. 2008;29:608-615.

11. Hartnug H-P. Advances in the understanding of the mechanisms of IVIG. J Neurol. 2008;255:3-6.

12. Basta M. Ambivalent effect of immunoglobulin on the complement system: activation versus inhibition. Mol Immunol. 2008;45:4073-4079.

13. Montgomery RA, Zachary AA, Racusen LC, et al. Plasmapheresis and intravenous immune globulin provides effective rescue therapy for refractory humoral rejection and allows kidneys to be successfully transplanted into crossmatch positive recipients. Transplantation. 2000;70:887-895.

14. Montgomery RA, Lonze BE, King KE, et al. Desensitization in HLAincompatible kidney recipients and survival. $N$ Engl J Med. 2011;365: 318-326.

15. Vo AA, Peng A, Toyoda M, et al. Use of intravenous immune globulin and rituximab for desensitization of highly HLA-sensitized patients awaiting kidney transplantation. Transplantation. 2010;89: 1095-1102.

16. Jordan SC, Tyan D, Stablein D, et al. Evaluation of intravenous immunoglobulin as an agent to lower allosensitization and improve transplantation in highly sensitized adult patients with end-stage renal disease: report of the NIG IG02 trial. J Am Soc Nephrol. 2004;15:3256-3262.

17. Peng A, Vo A, Villicana R, et al. Long-term graft and patient outcomes in highly HLA-sensitized deceased donor kidney transplant recipients desensitized with high dose IVIG. Am J Transplant. 2008;8:303.

18. Stegall MD, Gloor J, Winters JL, Moore SB, Degoey S. A comparison of plasmapheresis versus high-dose IVIG desensitization in renal allograft recipients with high levels of donor specific alloantibody. Am J Transplant. 2006;6:346-351.

19. Marfo K, Ling M, Bao Y, et al. Lack of effect in desensitization with intravenous immunoglobulin and rituximab in highly sensitized patients. Transplantation. 2012;94:345-351.

20. Lobashevsky AL, Higgins NG, Rosner KM, Mujtaba MA, Goggins WC, Taber TE. Analysis of anti-HLA antibodies in sensitized kidney transplant candidates subjected to desensitization with intravenous immunoglobulin and rituximab. Transplantation. 2013;96:182-190.

21. Vo AA, Lukovsky M, Toyoda M, et al. Rituximab and intravenous immune globulin for desensitization during renal transplantation. N Engl J Med. 2008;359:242-251.

22. Vo AA, Petrozzino J, Yeung K, et al. Efficacy, outcomes, and cost-effectiveness of desensitization using IVIG and rituximab. Transplantation. 2013;95:1-7.

23. Zachary AA, Montgomery RA, Leffel MS. Factors associated with and predictive of persistence of donor-specific antibody after treatment with plasmapheresis and intravenous immunoglobulin. Hum Immunol. 2005;66:364-370.

24. Cecka JM, Zhang Q, Reed EF. Preformed cytotoxic antibodies in potential allograft recipients: recent data. Hum Immunol. 2005;66: 343-349.

25. Reinsmoen NL, Lai CH, Vo A, et al. Acceptable donor-specific antibody levels allowing for successful deceased and living donor kidney transplantation after desensitization therapy. Transplantation. 2008;86: 820-825.

26. Yabu JM, Higgins JP, Chen G, et al. C1q-fixing human leukocyte antigen antibodies are specific for predicting transplant glomerulopathy and late graft failure after kidney transplantation. Transplantation. 2011;91: 342-347.

27. US Renal Data System. USRDS 2008 Annual Data Report: Atlas of Chronic Kidney Disease and End-Stage Renal Disease in the United States. Bethesda, MD, USA: National Institutes of Health, National Institute of Diabetes and Digestive and Kidney Diseases; 2008. Available from: http://www.usrds.org/atlas08.aspx. Accessed August 20, 2014.

28. Shehata N, Palda VA, Meyer RM, et al. The use of immunoglobulin therapy for patients undergoing solid organ transplantation: an evidence-based practice guideline. Transfus Med Rev. 2010;24 Suppl 1: S7-S27.
29. Jordan SC, Vo AA, Peng A, Toyoda M, Tyan D. Intravenous gammaglobulin (IVIG): a novel approach to improve transplant rates and outcomes in highly HLA-sensitized patients. Am J Transplant. 2006;6: 459-466.

30. Jordan SC, Toyoda M, Kahwaji J, et al. Intravenous immunoglobulin (IVIG) a modular of immunity and inflammation with applications in solid organ transplantation. In: Kaplan B, Burkhart GJ, Lakkis FG, editors. Immunotherapy in Transplantation: Principles and Practice. Oxford, UK: Wiley-Blackwell; 2012.

31. Gabardi S, Townsend K, Martin ST, Chandraker A. Evaluating the impact of pre-transplant desensitization utilizing a plasmapheresis and low-dose intravenous immunoglobulin protocol on BK viremia in renal transplant recipients. Transpl Infect Dis. 2013;15:361-368.

32. Kahwaji J, Sinha A, Toyoda M, et al. Infectious complications in kidney-transplant recipients desensitized with rituximab and intravenous immunoglobulin. Clin J Am Soc Nephrol. 2011;6:2894-2900.

33. Barbosa D, Kahwaji J, Puliyanda D, et al. Polyomavirus BK viremia in kidney transplant recipients after desensitization with IVIG and rituximab. Transplantation. November 21, 2013. [Epub ahead of print.]

34. Ulrich C, Kanitakis J, Stockfleth E, Euvrard S. Skin cancer in organ transplant recipients - where do we stand today? Am J Transplant. 2008;8:2192-2198.

35. Kaplan B, Gngemi A, Thielke J, Oberholzer J, Sankary H, Benedetti E. Successful rescue of refractory, severe antibody-mediated rejection with splenectomy. Transplantation. 2007;83:99-100.

36. Locke JE, Zachary AA, Haas M, et al. The utility of splenectomy as rescue treatment for severe acute antibody-mediated rejection. $\mathrm{Am} \mathrm{J}$ Transplant. 2007;7:842-846.

37. Cecka JM, Kucheryavaya AY, Reinsmoen NL, Leffell MS. Calculated PRA: initial results show benefits for sensitized patients and a reduction in positive crossmatches. Am J Transplant. 2011;11:719-724.

38. Israni AK, Salkowski N, Gustafson S, et al. New national allocation policy for deceased donor kidneys in the United States and possible effect on patient outcomes. J Am Soc Nephrol. 2014;25: $1842-1848$

39. Locke JE, Gentry SE, Simpkins CE, et al. Combining kidney paired donation, desensitization, and non-directed donors to transplant multiple highly sensitized patients. Am J Transplant. 2006;6:808.

40. Yabu JM, Pando MJ, Busque S, Melcher ML. Desensitization combined with paired exchange leads to successful transplantation in highly sensitized kidney transplant recipients: strategy and report of five cases. Transplant Proc. 2013;45:82-87.

41. Vo AA, Wechsler EA, Wang J, et al. Analysis of subcutaneous (SQ) alemtuzumab induction therapy in highly sensitized patients desensitized with IVIG and rituximab. Am J Transplant. 2008;8:144-149.

42. Everly MJ, Everly JJ, Susskind B, et al. Bortezomib provides effective therapy for antibody- and cell-mediated acute rejection. Transplantation. 2008;86:1754-1761.

43. Wu G, Chai N, Kim I, Klein AS, Jordan SC. Monoclonal antiinterleukin 6 receptor antibody attenuates donor-specific antibody responses in a mouse model of allosensitization. Transpl Immunol. 2013;28:138-143.

44. Kim I, Wu G, Chia N, et al. Anti-IL-6 receptor antibody attenuates recall antibody responses in a mouse model of allosensitization. Transplantation. In press 2014.

45. Chan A, Carter P. Therapeutic antibodies for autoimmunity and inflammation. Nat Rev Immunol. 2010;10:301-316.

46. Mel H, Schmidt S, Dorner R. Rationale of anti-CD19 immunotherapy: an option to target autoreactive plasma cells in autoimmunity. Arthritis Res Ther. 2012;14 Suppl 5:S1. 


\section{Publish your work in this journal}

Transplant Research and Risk Management is an international, peerreviewed open access journal focusing on all aspects of transplantation and risk management to achieve optimal outcomes in the recipient improving survival and quality of life. The journal welcomes submitted papers covering original research, basic science, clinical studies, reviews \& evaluations, guidelines, expert opinion and commentary, case reports and extended reports. The manuscript management system is completely online and includes a very quick and fair peer-review system, which is all easy to use. Visit http://www.dovepress.com/ testimonials.php to read real quotes from published authors.

Submit your manuscript here: http://www.dovepress.com/transplant-research-and-risk-management-journal 